\title{
A Soft Computing System for Modelling the Manufacture of Steel Components
}

\author{
Andres Bustillo, Javier Sedano, Leticia Curiel, José R. Villar and Emilio Corchado
}

\begin{abstract}
In this paper we present a soft computing system developed to optimize the laser milling manufacture of high value steel components, a relatively new and interesting industrial technique. This multidisciplinary study is based on the application of neural projection models in conjunction with identification systems, in order to find the optimal operating conditions in this industrial issue. Sensors on a laser milling centre capture the data used in this industrial case study defined under the frame of a machine-tool that manufactures steel components like high value molds and dies. The presented model is based on a two-phase application. The first phase uses a neural projection model capable of determine if the data collected is informative enough based on the existence of internal patterns. The second phase is focus on identifying a model for the laser-milling process based on low-order models such as Black Box ones. The whole system is capable of approximating the optimal form of the model. Finally, it is shown that the Box-Jenkins algorithm, which calculates the function of a linear system from its input and output samples, is the most appropriate model to control such industrial task for the case of steel components.
\end{abstract}

\section{Introduction}

Laser milling, in general, consists on the controlled evaporation of waste material due to its interaction with high-energy pulsed laser beams. The operator of a con-

Andres Bustillo, Leticia Curiel and Emilio Corchado

Department of Civil Engineering, University of Burgos, Burgos (Spain), e-mail: \{abustillo, lcuriel, escorchado\}@ubu.es

Javier Sedano

Department of Electromechanical Engineering. University of Burgos, Burgos (Spain), e-mail: jsedano@ubu.es

José R. Villar

Department of Computer Science, University of Oviedo (Spain), e-mail: villarjose@ uniovi.es 
ventional milling machine is aware at all times of the amount of waste material removed, but the same can not be said of a laser milling machine. Then a soft computing model that could predict the exact amount of material that each laser pulse is able to remove would contribute to the industrial use and development of this new technology. In this case we are focus on laser milling of steel components. It is an especially interesting industrial process, due to the broad use of steel as base material for different kind of manufacture tools, like molds and dies. One of the applications of this technology to these industrial tools is the deep indelible engraving of serial numbers or barcodes for quality control and security reasons for automotive industry [1]. The soft computing model proposed in this paper is able to optimize the manufacturing process and to control laser milling to the level of accuracy, productivity and surface quality that are required for the manufacture of deep indelible engravings.

The rest of the paper is organized as follows. Following the introduction, a twophase process is described to identify the optimal conditions for the industrial laser milling of steel components. The case study that outlines the practical application of the model is then presented. Finally, some different modelling systems are applied and compared, in order to select the optimal model, before ending with some conclusions and future work.

\section{An Industrial Process for Steel Components Modelling}

\subsection{Analyse the Internal Structure of the Data Set}

Cooperative Maximum-Likelihood Hebbian Learning (CMLHL) [2] is used in order to analyse the internal structure of the data set, which describe a steel manufactured component to establish whether it is sufficiently informative. CMLHL is a Exploratory Projection Pursuit (EPP) method $[3,4,5]$. EPP provides a linear projection of a data set, but it projects the data onto a set of basic vectors which help to reveal the most interesting data structures; interestingness is usually defined in terms of how far is the distribution from the Gaussian distribution [6]. MaximumLikelihood Hebbian Learning (MLHL) [5, 7] identifies interestingness by maximising the probability of the residuals under specific probability density functions that are non-Gaussian. An extended version is the CMLHL [2] model, which is based on MLHL [5, 7] but adds lateral connections [8, 2] that have been derived from the Rectified Gaussian Distribution [6]. Considering an N-dimensional input vector $(\bar{x})$, and an M-dimensional output vector $(\bar{y})$, with $\bar{W}_{i j}$ being the weight (linking input $j$ to output $i$ ), then CMLHL can be expressed $[9,10]$ as:

$$
\text { Feed-forward step : } y_{i}=\sum_{j=1}^{N} W_{i j} \times x_{j}, \forall i
$$




$$
\begin{array}{r}
\text { Lateral activation passing : } y_{i}(t+1)=\left[y_{i}(t)+\delta(b-A \times y)\right] \\
\text { Feedback step }: e_{j}=x_{j}-\sum_{i=1}^{M} W_{i j} \times y_{i}, \forall j, \\
\text { Weight change }: \Delta W_{i j}=\eta \times y_{i} \times \operatorname{sign}\left(e_{j}\right) \times\left|e_{j}\right|^{p-1}
\end{array}
$$

Where $\eta$ is the learning rate, $\tau$ is the strength of the lateral connections, $b$ the bias parameter, $p$ a parameter related to the energy function $[2,5,7]$ and $A$ a symmetric matrix used to modify the response to the data [2]. The effect of this matrix is based on the relation between the distances separating the output neurons.

\subsection{The identification criterion}

The identification criterion evaluates which of the group of candidate models is best adapted to and which best describes the data sets collected in the experiment; i.e., given a model $M\left(\theta_{*}\right)$ its prediction error may be defined by equation (5); and a good model [9] will be that which makes the best predictions, and which produces the smallest errors when compared against the observed data. In other words, for any given data group $Z^{t}$, the ideal model will calculate the prediction error $\varepsilon\left(t, \theta_{N}\right)$, equation (5), in such a way that for any one $t=N$, a particular $\hat{\theta}_{*}$ (estimated parametrical vector) is selected so that the prediction error $\varepsilon\left(t, \hat{\theta}_{N}\right)$ in $t=1,2, \ldots, N$, is made as small as possible.

$$
\varepsilon\left(t, \theta_{*}\right)=y(t)-\hat{y}\left(t \mid \theta_{*}\right) .
$$

The estimated parametrical vector $\hat{\theta}$ that minimizes the error, equation (8), is obtained from the minimization of the error function (6). This is obtained by applying the least-squares criterion for the linear regression, i.e., by applying the quadratic norm $\ell=\frac{1}{2} \varepsilon^{2}$, equation (7).

$$
\begin{array}{r}
V_{N}\left(\theta, Z^{N}\right)=\frac{1}{N} \sum_{i=1}^{N} \ell\left(\varepsilon_{F}(t, \theta)\right) \\
V_{N}\left(\theta, Z^{N}\right)=\frac{1}{N} \sum_{t=1}^{N} \frac{1}{2}(y(t)-\hat{y}(t \mid \theta))^{2} \\
\hat{\theta}=\hat{\theta}_{N}\left(Z^{N}\right)=\operatorname{argmin}_{\theta \in D_{M}} V_{N}\left(\theta, Z^{N}\right)
\end{array}
$$

The methodology of black-box structures has the advantage of only requiring very few explicit assumptions regarding the pattern to be identified, but that in turn makes it difficult to quantify the model that is obtained. The discrete linear models may be represented through the union between a deterministic and a stochastic part, equation (9); the term $e(t)$ (white noise signal) includes the modelling errors and is associated with a series of random variables, of mean null value and variance $\lambda$. 


$$
y(t)=G\left(q^{-1}\right) \times u(t)+H\left(q^{-1}\right) \times e(t)
$$

The structure of a black-box model depends on the way in which the noise is modelled $H\left(q^{-1}\right)$; thus, if this value is 1, then the OE (Output Error) model is applicable; whereas, if it is different from zero a great range of models may be applicable; one of the most common being the BJ (Box Jenkins) algorithm. This structure may be represented in the form of a general model, where $B\left(q^{-1}\right)$ is a polynomial of grade $n_{b}$, which can incorporate pure delay $n_{k}$ in the inputs, and $A\left(q^{-1}\right), C\left(q^{-1}\right)$, $D\left(q^{-1}\right)$ and $F\left(q^{-1}\right)$ are autoregressive polynomials ordered as $n_{a}, n_{c}, n_{d}, n_{f}$, respectively (10). Likewise, it is possible to use a predictor expression, for the on-step prediction ahead of the output $y(t \mid \theta)(11)$. Polynomials used in (10) are B, F and B, $\mathrm{F}, \mathrm{C}$ and $\mathrm{D}$ for OE and BJ models, respectively.

$$
\begin{array}{r}
A\left(q^{-1}\right) \times y(t)=q^{-n_{k}} \times \frac{B\left(q^{-1}\right)}{F\left(q^{-1}\right)} \times u(t)+\frac{C\left(q^{-1}\right)}{D\left(q^{-1}\right)} \times e(t) \\
\hat{y}(t \mid \theta)=\frac{D\left(q^{-1}\right) \times B\left(q^{-1}\right)}{C\left(q^{-1}\right) \times F\left(q^{-1}\right)} \times u(t)+\left[1-\frac{D\left(q^{-1}\right) \times A\left(q^{-1}\right)}{C\left(q^{-1}\right)}\right] \times y(t)
\end{array}
$$

Procedure for Modelling the Laser Milling Process. The identification procedure is carried out in accordance with two fundamental patterns: a first pre-analytical and then an analytical stage that assists with the determination of the parameters in the identification process and the model estimation. The pre-analysis test is run to establish the identification techniques [9, 10,11,12], the selection of the model structure and its order estimation [13, 14], the identification criterion and search methods that minimize it and the specific parametrical selection for each type of model structure. A second validation stage ensures that the selected model meets the necessary conditions for estimation and prediction. Three tests were performed to validate the model: residual analysis $\varepsilon(t, \hat{\theta}(t))$, by means of a correlation test between inputs, residuals and their combinations; final prediction error (FPE) estimate, as explained by Akaike [15]; and the graphical comparison between desired outputs and the outcome of the models through simulation one (or k) steps before.

\section{Modelling Steel Components: An Industrial Task}

This research is interested on the study and identification of the optimal conditions for laser milling of deep indelible engraving of serial numbers or barcodes on steel components using a commercial Nd:YAG laser with a pulse length of $10 \mu \mathrm{s}$. Three parameters of the laser process can be controlled: laser power $\left(u_{1}\right)$, laser milling speed $\left(u_{2}\right)$ and laser pulse frequency $\left(u_{3}\right)$. The laser is integrated in a laser milling centre (DMG Lasertec 40). 
To simplify this industrial problem a test piece was designed and used in all of the laser milling experiments. It consisted on an inverted, truncated, pyramid profile that had to be laser milled on a flat metallic piece of steel. The truncated pyramid had angles of $135^{\circ}$, and a depth of $1 \mathrm{~mm}$, but as the optimized parameters for the laser milling of steel were not known at that point in time, both parameters showed errors, which are referred as angle error $\left(y_{1}\right)$ and depth error $\left(y_{2}\right)$. A third parameter to be considered is the removal rate, that is, the volume of steel removed by the laser per minute $\left(y_{3}\right)$. A last parameter is the surface roughness of the milled piece $\left(y_{4}\right)$, measured on the flat surface of the truncated pyramid. These four variables have to be optimized, because the industrial process required a precise geometrical shape, the shorter manufacturing time and a good surface roughness of the piece. We applied different modelling systems to achieve the optimal conditions of these four parameters, although for demonstration we only show one of these four parameters.

Table 1 Variables, units and values used during the experiments. Output $y(t)$, Input $u(t)$.

\begin{tabular}{ll}
\hline Variable (Units) & Range \\
\hline Angle error of the test piece, $y_{1}(t)$ & -1 to 1 \\
Depth error of the test piece, $y_{2}(t)$ & -1 to 1 \\
Material removal rate $\left(\mathrm{mm}^{3} / \mathrm{min}\right), y_{3}(t)$ & 0.02 to 0,75 \\
Surface roughness of the test piece $(\mu \mathrm{m}), y_{4}(t)$ & 0.324 .38 \\
Laser power in percent of the maximum power performed by the laser $(\%)$, & 20 to 100 \\
$u_{1}(t)$ & 200 to 800 \\
Laser milling speed $(\mathrm{mm} / \mathrm{s}), u_{2}(t)$ & 20 to 100 \\
Laser pulse frequency $(\mathrm{kHz}), u_{3}(t)$ & \\
\hline
\end{tabular}

The experimental design was performed on a Taguchi L25 with 3 input parameters and 5 levels, so as to include the entire range of laser milling settings that are controllable by the operator. Table 1 summarizes the input and output variables of the experiment which define the case of study. After the laser milling, actual inverted pyramid depth, walls angle and surface roughness $\left(y_{3}\right)$ of the bottom surface were measured using optical devices. The material removal rate $\left(y_{4}\right)$ was calculated from the whole time required for the manufacture of each sample and the actual volume of removed material . The measured walls angle and the pyramid depth were compared with the nominal values in the CAD model, thereby obtaining the two errors $\left(y_{1}\right.$ and $\left.y_{2}\right)$. The test piece and the prototype were described in detail beforehand [16].

\subsection{Application of the two phases of the modelling system}

The study has been organized into two phases or steps: 
1. Analysis of the internal structure of the data set based on the application of several unsupervised connectionist models, CMLHL projections are used.

2. Application of several identification models in order to find the one that best defines the dynamic of the laser milling process.

Step 1. The CMLHL projections: Fig. 1 shows the results obtained by means of CMLHL projections. This model is able to identify five different clusters order mainly by power. After studying each cluster it can be noted a second classification based on the speed and frequency as it is shown in Fig. 1. All this indicates that the data analysed is sufficiently informative.

Step 2. Modelling the laser milling process: Fig. 2, shows the result of output $\mathrm{y} 1(\mathrm{t})$, angle error, for the different models. They show the graphic representations of the results, for $\mathrm{OE}$ and $\mathrm{BJ}$ models, in relation to the polynomial order and the delay in the inputs; various delays for all inputs and various polynomial orders $\left[n_{b 1}\right.$ $\left.\begin{array}{lllllllllll}n_{b 2} & n_{b 3} & n_{c} & n_{d} & n_{f} & n_{k 1} & n_{k 2} & n_{k 3}\end{array}\right]$ were considered to arrive at the highest degree of precision, in accordance with the structure of the models that have been used; see Table 1. In Fig. 2, the X-axis shows the number of samples used in the validation of the model, while the Y-axis represents the range of output variables.

Table 2 shows a comparison of the qualities of estimation and prediction of the best models obtained, as a function of the model, the estimation method, and the indexes, which are defined as follows:

- The percentage representation of the estimated model (expressed as so many percent \%) in relation to the true system: the normalized mean error that is computed with one-step, ten-steps prediction (FIT1 and FT10, respectively) or by means of simulation (FIT). The one-step and ten-steps predictions $\left(\hat{y}_{1}(t \mid m)\right.$ and $\left.\hat{y}_{10}(t \mid m)\right)$ and the model simulation $\hat{y}_{\infty}(t \mid m)$ are also shown.

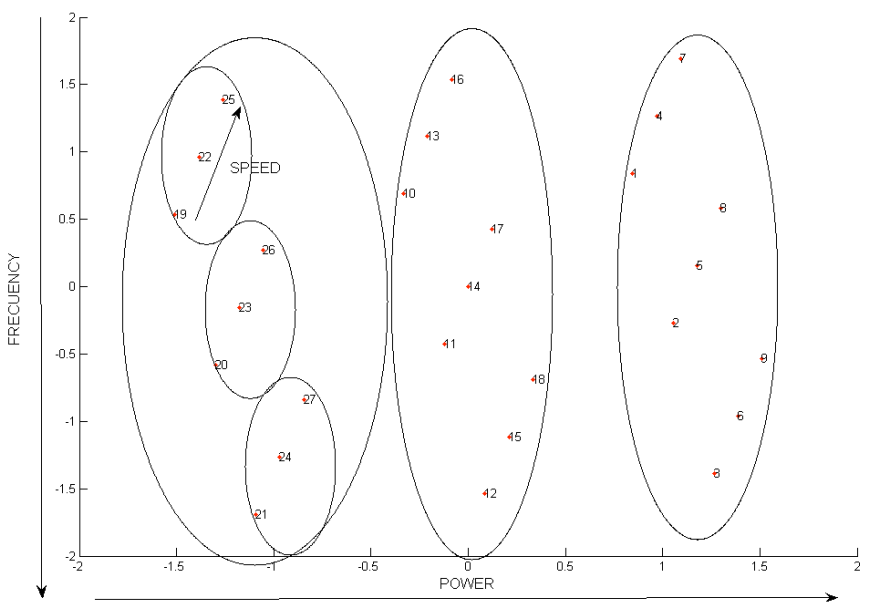

Fig. 1 The first of the two projections obtained by CMLHL 

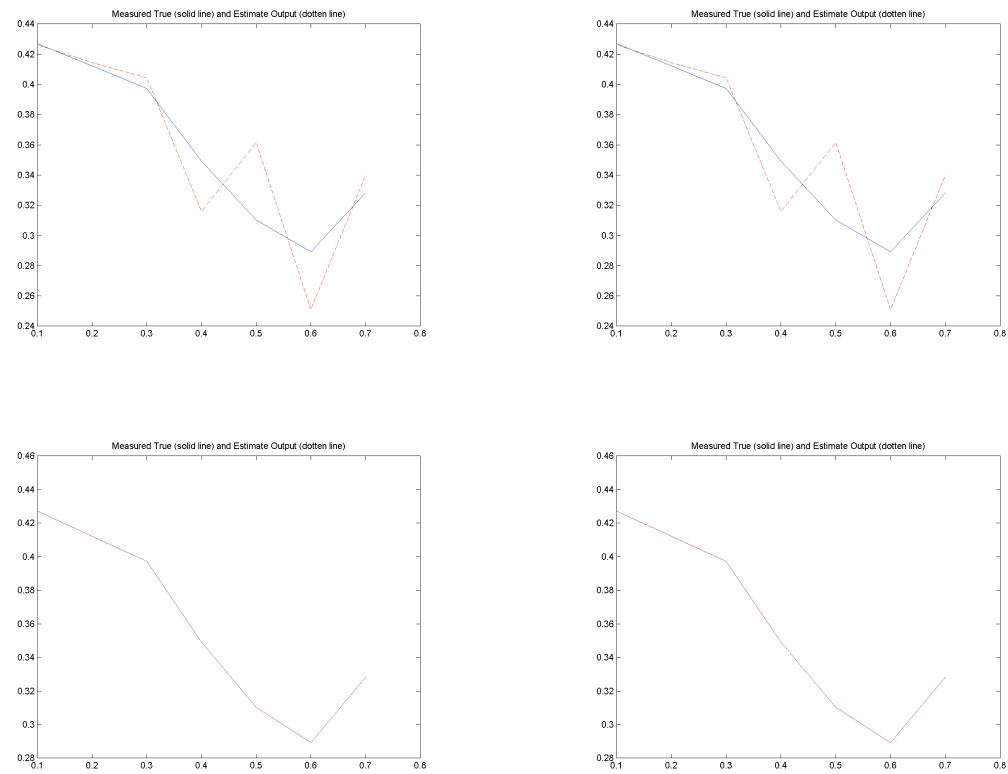

Fig. 2 Output response of two different models: the OE -upper row- and BJ -the lower row- methods. Left column figures represents the angle error output $y_{1}(t)$ simulation, while the right column figures correspond with the one step ahead prediction of the angle error output $y_{1}(t)$. The validation data set was not used for the estimation of the model. The order of the structure of the model

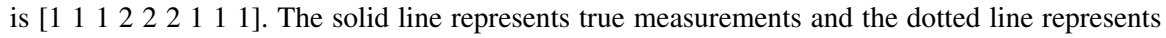
estimated output.

- The loss or the error function (V): the numeric value of the mean square error that is calculated from the estimation data set.

- The generalization error value (NSSE): the mean square error calculated with the validation data set.

- The average generalization error value (FPE): the FPE criterion calculated from the estimation data set.

From the graphical representation (Fig. 2) it can be concluded that the BJ model is capable of simulating and predicting the behaviour of the laser milled piece for angle error, as they meet the indicators and is capable of modelling more than $95 \%$ of the true measurements. The tests were performed using Matlab and the System Identification Toolbox. Table 3 shows the final BJ model. 
Table 2 Indicator values for several proposed models of the angle error

\begin{tabular}{|c|c|}
\hline Model & Indexes \\
\hline $\begin{array}{l}\text { Black-box OE model with } n_{b 1}=2, n_{b 2}=1, n_{b 3}=1, n_{c}=2, n_{d}=2 \text {, } \\
n_{f}=2, n_{k 1}=1, n_{k 2}=1, n_{k 3}=1 \text {. The model is estimated using the } \\
\text { prediction error method, the degree of the model selection is carried } \\
\text { out from the best AIC criterion (the structure that minimizes AIC). }\end{array}$ & $\begin{array}{l}\text { FIT: } 44.04 \%, \text { FIT } 1: 44.04 \% \\
\text { FIT10:44.04\%, V: } 0.02 \\
\text { FPE:0.23, NSSE:7.71e-4 }\end{array}$ \\
\hline $\begin{array}{l}\text { Black-box OE model } n_{b 1}=1, n_{b 2}=1, n_{b 3}=1, n_{c}=2, n_{d}=2 \text {, } \\
n_{f}=2, n_{k 1}=1, n_{k 2}=1, n_{k 3}=1 \text {.The model is estimated using the } \\
\text { prediction error method, the degree of the model selection is carried } \\
\text { out with the best AIC criterion (the structure that minimizes AIC). }\end{array}$ & $\begin{array}{l}\text { FIT: } 21.2 \%, \text { FIT1: } 21.2 \% \\
\text { FIT10: 21.2\%, V: } 0.023 \\
\text { FPE:0.162, NSSE:0.0015 }\end{array}$ \\
\hline $\begin{array}{l}\text { Black-box BJ model with } n_{b 1}=2, n_{b 2}=1, n_{b 3}=1, n_{c}=2, n_{d}=2 \text {, } \\
n_{f}=2, n_{k 1}=1, n_{k 2}=1, n_{k 3}=1 \text {. The model is estimated using the } \\
\text { prediction error method, the degree of the model selection is carried } \\
\text { out with the best AIC criterion (the structure that minimizes AIC). }\end{array}$ & $\begin{array}{l}\text { FIT: } 100 \%, \quad \text { FIT1: } 100 \% \\
\text { FIT10:100\%, } \quad \text { V: } \quad 0.12 \\
\text { FPE:0.27, NSSE:2.73e-31 }\end{array}$ \\
\hline $\begin{array}{l}\text { Black-box BJ model with } n_{b 1}=1, n_{b 2}=1, n_{b 3}=1, n_{c}=2, n_{d}=2 \text {, } \\
n_{f}=2, n_{k 1}=1, n_{k 2}=1, n_{k 3}=1 \text {. The model is estimated using the } \\
\text { prediction error method, the degree of the model selection is carried } \\
\text { out with the best AIC criterion (the structure that minimizes AIC). }\end{array}$ & $\begin{array}{l}\text { FIT: } 100 \%, \quad \text { FIT1: } 100 \% \\
\text { FIT10:100\%, V: } 0.97 \\
\text { FPE: } 1,75, \text { NSSE:4.17e-30 }\end{array}$ \\
\hline
\end{tabular}

Table 3 Function and parameters that represent the behaviour of the laser milled piece for the angle error. The degree of the $\mathrm{BJ}$ model polynomials are $n_{b 1}=1, n_{b 2}=1, n_{b 3}=1, n_{c}=2, n_{d}=$

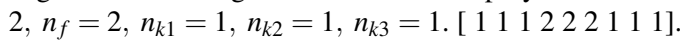

\begin{tabular}{ll}
\hline Final polynomials. & \\
\hline$B 1(q)=0.01269 \times q^{-1}$ & $D(q)=1+1.208 \times q^{-1}+0.3098 \times q^{-2}$ \\
$B 2(q)=0.0004895 \times q^{-1}$ & $F 1(q)=1+0.4094 q^{-1}-0.16 \times q^{-2}$ \\
$B 3(q)=0.01366 \times q^{-1}$ & $F 2(q)=1-1.678 \times q^{-1}+0.7838 \times q^{-2}$ \\
$C(q)=1+1.541 \times q^{-1}+1.02 \times q^{-2}$ & $F 3(q)=1-1.1 \times q^{-1}+0.7671 \times q^{-2}$ \\
& $\mathrm{e}(\mathrm{t})$ is white noise signal whit variance 0.08 \\
\hline
\end{tabular}

\section{Conclusions and Futures lines of Work}

We have done an investigation to study and identify the most appropriate modelling system for laser milling of steel components. Several methods were investigated to achieve the best practical solution to this interesting problem. The study shows that the Box-Jenkins algorithm is best adapted to this case, in terms of identifying the best conditions and predicting future circumstances. It is important to emphasize that a relevant aspect of this research lies in the use of a two-step model when modelling the laser milling process for steel components: a first step, which applies projection methods to establish whether the data describing the case study is sufficiently informative. As a consequence, the first phase eliminates one of the problems associated with these identification systems, which is that of having no prior knowledge of whether the experiment that generated the data group may be considered acceptable and will present sufficient information in order to identify the overall nature of the problem. 
Future work will be focus on the study and application of this model to other kinds of materials of industrial interest, such as cast single-crystal nickel superalloys for high-pressure turbine blades and also the application of this model to the optimization of different but similar industrial problems, like laser cladding, laser super-polishing and laser drilling.

Acknowledgements Thanks to the support received from ASCAMM Technological Centre http://www.ascamm.com-, which provided the laser milling data and performed all the laser tests. The authors would especially like to thank Mr. P. Palouzie and Mr. J. Diaz for their kind-spirited and useful advice. This research has been partially supported through project BU006A08 of JCyL and through project CIT-020000-2008-2 of Spanish Ministry of Education and Innovation.

\section{References}

1. Wendland, J., Harrison, P.M., Henry, M., Brownell, M.: Deep Engraving of Metals for the Automotive Sector Using High Average Power Diode Pumped Solid State Lasers, Proceedings of the 23nd International Conference on Applications of Lasers and Electro-Optics (ICALEO 2005)

2. Corchado, E., Fyfe, C.: Connectionist Techniques for the Identification and Suppression of Interfering Underlying Factors, J. of Pattern Recognition and Artificial Intelligence 17, 8, 1447-1466 (2003)

3. Diaconis, P., Freedman, D.: Asymptotics of Graphical Projections, The Annals of Statistics 12, 3, 793-815 (1984)

4. Friedman, J. H., Tukey, J.W.: Projection Pursuit Algorithm for Exploratory Data-Analysis, IEEE Transactions on Computers 23, 9, 881-890 (1974)

5. Corchado, E., MacDonald, D., Fyfe, C.: Maximum and Minimum Likelihood Hebbian Learning for Exploratory Projection Pursuit, Data Mining and Knowledge Discovery 8, 3, 203-225 (2004)

6. Seung, H.S., Socci, N.D., Lee, D.: The Rectified Gaussian Distribution, Advances in Neural Information Processing Systems 10, 350-35 (1998)

7. Fyfe, C., Corchado, E.: Maximum Likelihood Hebbian Rules, Proc. of the 10th European Symposium on Artificial Neural Networks (ESANN 2002)

8. Corchado, E., Han, Y., Fyfe, C.: Structuring Global Responses of Local Filters Using Lateral Connections, Journal of Experimental \& Theoretical Artificial Intelligence 15, 4, 473-487 (2003)

9. Ljung, L.: System Identification, Theory for the User, Prentice-Hall (1999)

10. Nögaard, N., Ravn, O., Poulsen, N.K., Hansen, L.K.: Neural Networks for Modelling and Control of Dynamic Systems, Springer-Verlag (2000)

11. Nelles, O.: Nonlinear System Identification, From Classical Approaches to Neural Networks and Fuzzy Models, Springer (2001)

12. Haber, R., Keviczky, L.: Nonlinear System Identification, Input-Output Modeling Approach, Part. 1: Nonlinear System Parameter Estimation, Kluwer Academic Publishers (1999)

13. Stoica, P., Söderström, T.: A useful parametrization for optimal experimental design, IEEE Trans. Automatic. Control AC-27 (1982)

14. He, X., Asada, H.: A new method for identifying orders of input-output models for nonlinear dynamic systems, Proceedings of the American Control Conference (1993)

15. Akaike, H.: Fitting autoregressive models for prediction, Ann. Inst. Stat. Math 20, 425-439, 1969

16. Arias, G., Ciurana, J., Planta, X., Crehuet, A.: Analyzing Process Parameters that influence laser machining of hardened steel using Taguchi method, Proceedings of 52nd International Technical Conference SAMPE 2007 